\title{
Design and Implementation of an Intelligent Remote Centre Compliance (IRCC) as a means of intelligent position feedback for a construction robot.
}

\author{
Glen Bright \\ Department of Mechatronics and Robotics \\ Mechanical Engineering \\ University of Natal \\ Durban \\ Brightg@ieng.und.ac.za \\ Craig Deubler \\ Department of Mechatronics and Robotics \\ Mechanical Engineering \\ University of Natal \\ Durban \\ deublercaenz.und.ac.za
}

\section{$\underline{\text { 1. Abstract }}$}

An intelligent Remote Centre Compliance (IRCC) system integrates manipulator position sensors into the robots control routine. The feedback provided gives the robot the ability to react to its environment. With the improvement in the robot's ability to interact with its surroundings, the manipulator becomes self-aware making it more efficient by providing a means of self-optimisation. In a construction site environment, where man and machine have to work in close proximity, safety is also improved significantly by robot self-awareness.

The IRCC is also used as a design tool to develop unique and specialised compliance devices, which have deformation characteristics optimised for particular tasks, which improves manipulator efficiency. This paper details the design of such an IRCC system for a construction robot.

\section{Introduction}

Robots are not widely used in the construction industry, as a construction site environmont is not as controlled or regular as an assembly plant. However, repetitive tasks such as riveting, welding or bricklaying are more suited to the use of industrial robots. Using robots to perforin these tasks allows site managers to allocate their human resources to more complex tasks.

Misalignment of mating parts is a significant problem in construction, due to factors such as part movement during assembly and inconsistency of the workpiece geometry i.e. Girders, rivets, bricks etc.
In such cases, selection of a suitable robot is not always easy, nor is programming it for optimal performance. Remote Centre Compliances (RCC's) are often used to reduce assembly difficulties, but in many instances more expensive, more advanced robots may be installed, in order to improve efficiency.

This project involves the development of an IRCC system that is integrated into a robots control system. This device improves the level of process monitoring, dynamic control and self-awareness to the robot. This enables the manipulator system to perform continuous quality control. The robot can continuously make minor corrections, which reduce the need for an opcrator to be present at all times. This puts existing equipment to better use, reduces stoppages and reduces the downtime required to reprogram manipulators on site.

The IRCC uses common industrial electronics to bring a simple robot up to state of the art intelligence capabilities. The system is used to investigate complex assembly problems such as assembling non-spherical parts or difficult insertions requiring large amounts of compliance. The data acquired is initially used to produce simplified routines and improve the cfficiency of such assemblies.

The data accumulated from the IRCC will be used to generate a detailed kincmatic model of these assemblies, which can be optimised for the assembly operation. The model will be used to develop a simple task specific attachment, a 'Task Optimised Compliance'. This will replace, or assist, an $\mathrm{RCC}$ in these tasks, making them faster and more efficient. 


\section{The need for compliance}

\subsection{Automated assembly}

During automated assembly tasks non-ideal geometric relationships between the mating parts and between the robot and assembly parts could cause severe problems. This will cause unsuccessful assembly, and in some cases damage to the workpieces.

This phenomenon has been extensively researched. Jamming and wedging are the two main conditions that cause unsuccessful part mating. [1]

\subsection{The Remote Centre Compliance mechanism}

A Remote Centre Compliance (RCC), is a mechanism described as a general error absorbing interface between the workpicce and the tool. This is a mechanism with the particular force deformation behaviour that we desire and was invented by Samucl Drake and Sergio Simunivic in 1976/7. [1]

The RCC is a simple passive device that operates on geometric principles An RCC corrects lateral misalignment by using the deformation characteristics of a parallelogram. Angular misalignment is corrected by using the kinematics of a trapezoid to rotate the workpiece around a, a remote centre of rotation.

As shown in Figure 1.

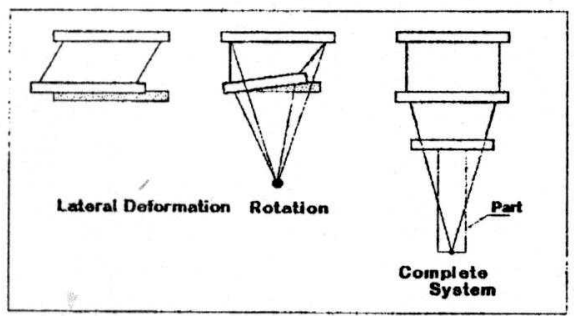

Figure1: RCC geometry

Industrial RCC's combine the lateral and trapezoidal deformation by using three Elastomer Shear Pad compliance members. These layered stacks of Elastomer and steel are compliant laterally but only slightly compliant axially. This slight axial compliance is sufficient to provide enough lateral deformation as not to warrant separate lateral members.

The RCC was used as a basis for this project as, being a completely passive device, it is a self contained 'problem solver' [refer to Figure 2]. A Force/Torque sensor or a vision system requires large amounts of computational analysis and controller feedback. The RCC will solve misalignments without any additional assistance. This characteristic of the RCC makes it a popular device in industry. The IRCC system monitors the movement of the $\mathrm{RCC}$ during asscmbly. The data gathered is used to generate a model of the assembly. The model facilitates the development of solutions to problems associated with the assembly routine. [See section 5]

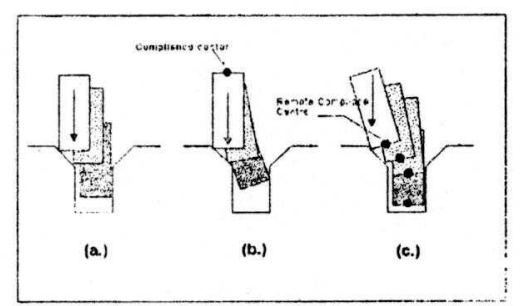

Figure 2 : Rotation about a remote centre

\section{The Intelligent Remote Centre Compliance}

Instrumenting an RCC is not a new idea. Draper Laboratories in the USA developed a similar device in the 19\%()s using a series of three 2 dimensional infrared positioning arrays. This approached was deemed unsuitable so more readily available industrial electronics have been used. [3]

\subsection{RCC position tracking}

The movement of the RCC is calculated by measuring the position of the lower plate of the RCC with respect to the top plate.

The mathematics used to calculatc the deformation of the IRCC maps three points on the lower plate of a 3 flexure RCC [see Figure 3.1. This gives the exact position of the lower plate, which is represented as a triangular laminar in the model. Each point is calculated by triangulation of its position with respect to the top plate of the RCC, resulting in nine degrees of freedom (d.o.f.). The three extra degrees of freedom give a level of redundancy that makes the system more computationally stable. The amount of data processing is also reduced by only having to calculate similar systems in 3 variables, as opposed to one large system with six variables

\subsection{Sensors}

Instrumenting the IRCC presented difficulties due to the small size of the device. The sensors had to precise, small and lightweight.

The space in which to install the sensors was limited when compared to the size of available sensors. Potential choices for sensors were LVDT's (lincar variable differential transformers) or cable extension potentiometers. LVDT's are precise, but difficult to install. Due to size constraints, the entire LVDT would have to be mounted externally.

The IRCC was constructed using custom-built cable extension potentiometer arrays. These sensors can be 
mounted externally and the cables run into the RCC. Furthermore potentiometers are inexpensive and readily available.

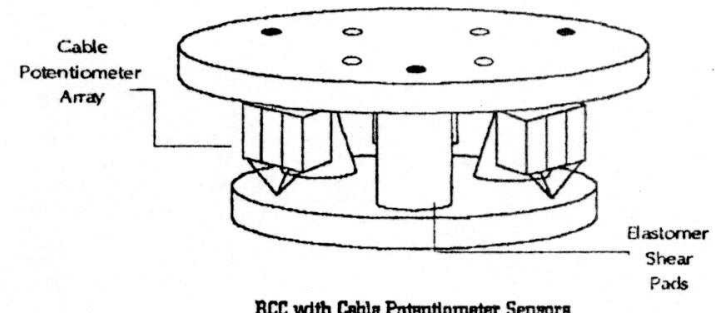

Figure 3: IRCC Schematic

\section{Data Processing}

The IRCC uses the data collected from cable potentiometers to develop models and algorithms that describe, in detail, the compliant movement of the RCC.

These models are processed into manipulator path corrections that optimise the assembly routine. [As shown in Figure 4]

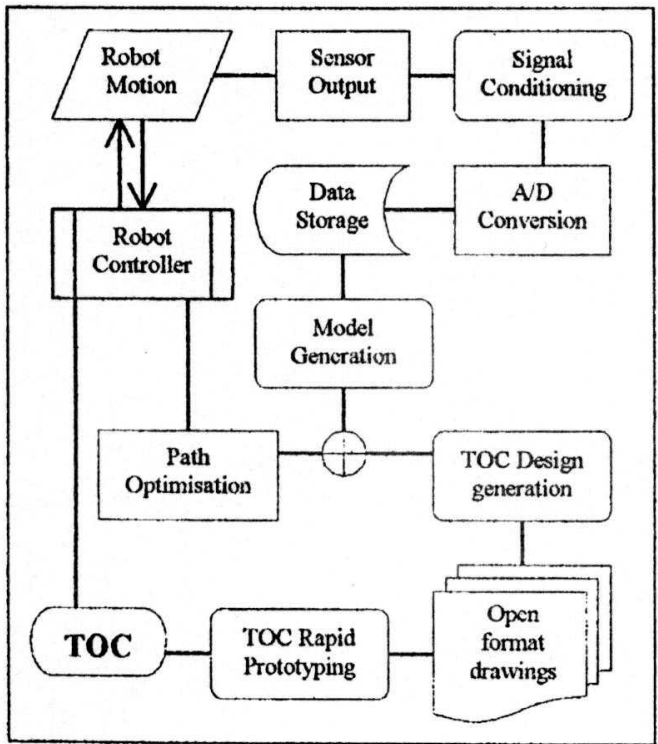

Fiqure 4: IRCC Data Processing

Where applicable, the assembly routine can be further optimised by developing a new compliance device to supplement or replace an RCC. The ability to develop the solid model of such a device is an integral part of the IRCC's smart systems.

\subsection{Signal Processing}

The IRCC system converts the resistance measured across each potentiometer into a normalised voltage signal by means of a bridge circuit. The voltages signals are filtered for noise then read into the controller by an A/D card.
Signal integrity must be preserved for accurate measurements. The signal path is shown in Figure 4

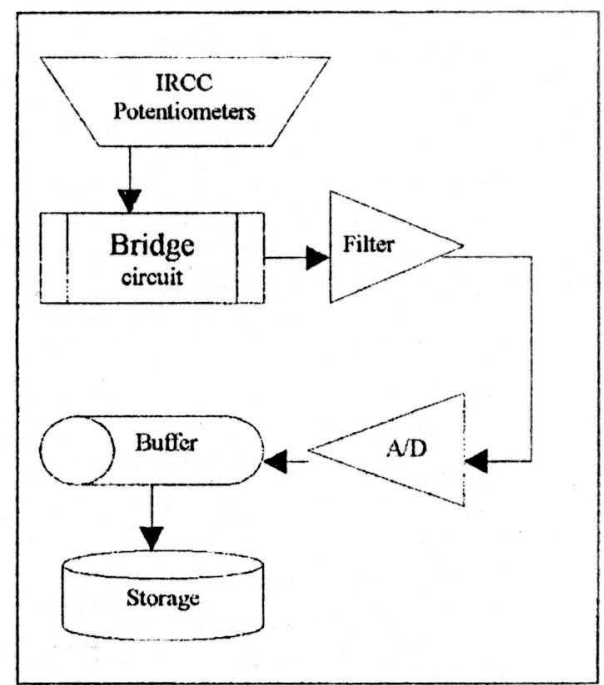

Figure 5: IRCC Signal Processing

\subsection{Data Acquisition}

Data is accumulated in a memory buffer before being stored. This incoming data is monitored for sudden changes in the RCC position, which is a safety feature protecting the robot and people working near it.

Due to the speed the RCC moves at and the small displacements experienced, accuracy and precision are required at this critical stage.

\subsection{Data Analysis}

The IRCC records systematic and random errors that deviate from the predicted workpiece path. Incorrectly placed parts or possible collisions with foreign bodies cause most random crrors. In such a case the IRCC acts as an active safety device, shutting down the system to prevent harm to personnel and damage to cquipment.

The data acquired during assembly is processed and examined for rcoccurring deformations in the RCC's motion. These errors show systematic inefficiencies in the manipulators assembly control program. The IRCC system analyses these errors and recalibrates the manipulator path to decrease cycle time and improve task efficiency.

The IRCC system maximises the speed of the robot by improving movement distribution between all available degrees of freedom in the manipulator and removing any robot movement that is nonessential.

However, path correction alone may not be sufficient to optimise some tasks. If, after path optimisation, there is still a need for compliance the IRCC's smart systems can generate a task specific compliance model. This model represents the specific compliance needs of a particular assembly routine. The IRCC smart systems use this model to generate the physical designs of a 'Task Optimised Compliance: 


\subsection{Software}

On a PC controlled manipulator, the IRCC interfaces directly with the Robot controller but without using much processor bandwidih. This allows us to run the IRCC using the same PC controller as the manipulator, rather than having to running two separate machines, or high-end hardware. This is a significant consideration if the manipulator has to be mobile. A mobile translational riveting robot would be an example. In the case of a robot with a dedicated controller the IRCC system can be run on an external PC.

The robot for which the IRCC is designed was a Puma 560. This particular manipulator has undergone conversion to an Adaptive PC based modular robotic system for part assembly [2]. This robotic system allows controller software add-ons and upgrades, without having to physically modify the controller. The software uses an open architecture, which enables third parties to develop software plug-ins for the manipulators control software. The IRCC software is a series of these plug-in modules. The IRCC modules are designed to perform certain tasks depending on the status of the robot and the processor bandwidth available.

The following modules have been implemented:

\section{Module 1: Data acquisition}

Designed to use as little processor bandwidth as possible: This module collects position information from the IRCC while it is in use and disables the instrumentation when the IRCC is not in use. Module 1 includes safety features such as crash detcction.

\section{Module 2: Data processing}

This 'self awareness' module monitors the assembly routine for systematic errors and does simple path reprogramming, such as workpiece realignment, for quality control. This module runs whenever there is sufficient processor capacity, for example, when robot is in a Wait State.

\section{Module 3: Graphical output}

When the Robot is offline, this module produces animations of the IRCC's movement, providing better insight into the operation for the operator.

\section{Module 4: Optimisation}

This module follows the path routine of the manipulator. The 'Optimisation' module performs all calculations and reprogramming necessary to generate a kinematic model of the assembly routine and to optimise the robots path programming. It does so:

1. By optimising the spced of the manipulator.

2. By reducing the d.o.f. needed for the assembly operation.

3. Where applicable, using the smart systems, to develop a solid model of a "Task Optimised Compliance".

\section{Task Optimised Compliance}

The Task Optimised Compliance (TOC) is a device compliant only in the axis required by a specific assembly routine. The advantage is that the device is more rigid than a typical RCC, which tend to be prone to free play and 'wobble' if a manipulator moves too fast. The TOC is designed for a specific operation, unlike the 'generalised' design of an RCC, which makes the TOC more efficient.

The non-standard shape of the TOC makes it complicated to manufacture by conventional means. It is not cost effective to make dies and jigs for a one off design. Flexible manufacturing means have to be used to produce the TOC. The development of a suitable manufacturing process for the TOC is currently underway.

\section{Future Plans}

\subsection{Generalising the IRCC}

New developments include enabling the system to perform analysis for a completely different class of robot. (E.g. Using a Puma robot to do analysis for a SCARA or gantry type robot.) Other advances would involve the ability to plan optimal plant floor placement for the robot.

Integrating the IRCC with an image recognition system is not currently being researched, but will be done in the near future.

\section{Conclusion}

The IRCC is an effective means of introducing learning capabilities, to robot manipulators, by using available industrial electronics. This allows us to use robots for monotonous repetitive tasks and to allocate human resources to complex tasks. This helps significantly in reducing costs and better utilising the workforce.

Technically, its smart design capabilities and Rapid Prototyping technologies enable the IRCC to produce TOC devices that would not otherwise be viable. The cost of the IRCC system is significantly lower than the alternatives: a Force/Torque ring or vision rig. By removing the need for large computational facilities, the cost of the controller is also reduced.

A construction environment is not an ideal location for a robot manipulator to work in. However, the benefits they offer in safety and resource usage make it worthwhile to develop systems for construction. The IRCC is a simple but effective way to improve robot feasibility in the construction industry.

\section{Acknowledgements}

The authors would like to thank the University of Natal, Durban and the Foundation for Research and Development 
for their continued support of this project, financially and otherwise.

\section{References}

1. Daniel Whitney; Remote Center Compliance; The Encyclopaedia of Robotics; Wiley Interscience 1988

2. Bright G, Potgieter J; Adaptive PC based modular Robotic system for part assembly operations; Proceedings $6^{\text {th }}$ IASTED International Conference on Robotics \& Manufacturing, Banff, Canada 1998

3. T.L. De Fazio, D.S. Seltzer, D.E. The Instrumented Remote Center Compliance; ROBOT SENSORS vol. 2 : Tactile and non-vision sensors; IFS Ltd 1986

4. Obikawa, Yoshino, Matsumura, Sasahara, Shinozuka, Furusawa; Rapid Manufacturing by sheet steel lanination; Proceedings $14^{\text {th }}$ International Conference on Computer-Aided Production Engineering Tokyo Sept. 1998

5. Stadler; Analytical Robotics and Mechatronics; McGraw Hill USA 1995 